\title{
SUPER-WAVELETS VERSUS POLY-BERGMAN SPACES
}

\author{
LUIS DANIEL ABREU
}

\begin{abstract}
Motivated by potential applications in multiplexing and by recent results on Gabor analysis with Hermite windows due to Gröchenig and Lyubarskii, we investigate vector-valued wavelet transforms and vector-valued wavelet frames, which constitute special cases of super-wavelets, with a particular attention to the case when the analyzing wavelet vector is constituted by functions $\Phi_{n}$ such that $\mathcal{F} \Phi_{n}(t)=t^{\frac{1}{2}} l_{n}^{0}(2 t)$, where $l_{n}^{0}$ is a Laguerre function. We construct an isometric isomorphism between $L^{2}\left(\mathbb{R}^{+}, \mathbf{C}^{n}\right)$ and poly-Bergman spaces, with a view to relate the sampling sequences in the poly-Bergman spaces to the wavelet frames and super-frames with the windows $\Phi_{n}$. One of the applications of the theory is a proof that $b \ln a<2 \pi(n+1)$ is a necessary condition for the (scalar) wavelet frame associated to the $\Phi_{n}$ to exist. This is the first known result of this type outside the setting of analytic functions (the case $n=0$, which has been completely studied by Seip in 1993).
\end{abstract}

\section{INTRODUCTION}

1.1. Motivation. Over the recent years, an increasing attention has been given to the study of vector-valued versions of time-frequency and time-scale methods, driven in part by potential applications in multiplexing, an important method in telecommunications, computer networks and digital video.

Roughly speaking, "multiplexing" means: encoding $n$ independent signals $f_{k}$ as a single signal $\mathbf{f}$ which contains all the information of all of the signals $f_{k}$.

Of course, the signals can always be combined into a single one by simple superposition; what makes the problem nontrivial, from a mathematical point of view, is to assure that the superposition is done in such a way that, when required, each of the signals $f_{k}$ can be recovered from the multiplexed signal $\mathbf{f}$ (demultiplexing). A recent mathematical approach to this problem has been proposed by means of the theory of frames. A sequence of functions $\left\{e_{j}\right\}_{j \in I}$ is said to be a frame in a Hilbert space $H$ if there exist constants $A$ and $B$ such that, for every $f \in H$,

$$
A\|f\|_{H}^{2} \leq \sum_{j \in I}\left|\left\langle f, e_{j}\right\rangle\right|^{2} \leq B\|f\|_{H}^{2} .
$$

In 4, Balan has given an interpretation of standard multiplexing methods from the point of view of super-frames, which are vector-valued versions of frames (where the Hilbert space $H$ is $L^{2}\left(\mathbb{R}, \mathbb{C}^{n}\right)$ ). The concept of super-frames has been investigated from the perspective of functional analysis by Han and Larson in [17] and several results have been obtained concerning the possibility of extending a frame to a

Date: September, 2009.

Key words and phrases. polyanalytic functions, wavelet frames and super frames, wavelet transform, polyanalytic Bergman spaces.

This research was partially supported by $\mathrm{CMUC} / \mathrm{FCT}$ and $\mathrm{FCT}$ post-doctoral grant SFRH/BPD/26078/2005, POCI 2010 and FSE.. 
super-frame [13, something that, not surprisingly, usually requires oversampling by a rate equal to dimension $n$, which represents the number of multiplexed signals [10, [16.

Within super-frames, super-wavelets have received a particular attention in [17, 13 and [10. Alternative approaches to the construction of vector-valued wavelets have also been considered recently [7, 6]. In this paper we will provide yet another approach to the construction of vector-valued wavelets with emphasis in important special cases where, perhaps in a surprising manner, a new connection with complex analysis is revealed.

To better give a context to our work, let us review two fundamental ideas which have arisen recently in different mathematical communities.

The first fundamental idea comes from Gabor and complex analysis: if one considers the super-frames built from shifting and modulating a window with n Hermite functions, we are lead to a very structured situation, where an intriguing connection to complex analysis has been explored by Gröchenig and Lyubarskii in [16] and then in [1, where it is also shown that the vector valued Gabor representations with vectorial Hermite windows correspond to polyanalytic functions (we provide definitions some lines below), in much the same way the scalar Gabor representations with Gaussian correspond to analytic functions.

The second fundamental idea is implicit in the link between the results of Hutnik [20, 21] and of Vasilevski [31, given the intriguing ressenblance between the description of wavelet spaces and poly-Bergman spaces.

In the present paper, we describe a connection between vector-valued wavelets and polyanalytic functions, in analogy to the one existing for the Gabor case [1, [2]. Indeed, polyanalytic Bergman spaces are the function-theoretic analogues of wavelet spaces, when one chooses as analysing wavelet a certain vector defined in terms of Laguerre functions. We will use this setting to provide a theoretical solution of the problem of multiplexing and demultiplexing vectorial signals in $L^{2}\left(\mathbb{R}^{+}, \mathbb{C}^{n}\right)$. We remind that, by the Paley-Wiener theorem, this space is unitarily equivalent to the vectorial Hardy space on the upper-half plane, $H^{2}\left(\mathbf{U}, \mathbb{C}^{n}\right)$.

1.2. Description of the results. To describe our results we need some definitions.

Let $\mathbf{U}=\{z \in \mathbb{C}: \operatorname{Re} z \geq 0\}$ stand for the upper half plane. The polyanalytic Bergman space, $\mathbf{A}^{n}(\mathbf{U})$ (poly-Bergman space, for short), is constituted by the complex valued functions defined on the upper half plane and such that

$$
\left(\frac{d}{d \bar{z}}\right)^{n} f(z)=0 \text { and } \int_{\mathbf{U}}|f(z)|^{2} d z<\infty .
$$

The space $\mathbf{A}^{0}(\mathbf{U})=A(\mathbf{U})$ is the usual (analytic) Bergman space in the upper half plane.

The potential applications of polyanalytic Bergman spaces in multiplexing are suggested by the orthogonal decomposition of the space $\mathbf{A}^{n}(\mathbf{U})$ in subspaces called true polyanalytic Bergman spaces, which will be denoted by $\mathcal{A}^{k}(\mathbf{U})$ :

$$
\mathbf{A}^{n}(\mathbf{U})=\mathcal{A}^{0}(\mathbf{U}) \oplus \ldots \oplus \mathcal{A}^{n-1}(\mathbf{U}) .
$$

In face of this decomposition, if we start with a vector $\mathbf{f}=\left(f_{0}, \ldots, f_{n-1}\right) \in L^{2}\left(\mathbb{R}^{+}, \mathbf{C}^{n}\right)$ and find a unitary transform (call it $B e r^{n}$ ) such that

$$
f_{k} \in L^{2}\left(\mathbb{R}^{+}\right) \stackrel{B e r^{k}}{\rightarrow} F_{k} \in \mathcal{A}^{k}(\mathbf{U})
$$


then we can store all the $F_{k}$ 's into a single multiplexed function

$$
F=F_{0}+\ldots+F_{n-1} \in \mathbf{A}^{n}(\mathbf{U}) .
$$

Then, thanks to the orthogonal decomposition of $\mathbf{A}^{n}(\mathbf{U})$, it is possible to recover each of the $F_{k}$, projecting the multiplexed signal $F$ into $\mathcal{A}^{k}(\mathbf{U})$. This is possible to be done using the reproducing kernel $K^{k}(z, w)$ of the spaces $\mathcal{A}^{k}(\mathbf{U})$ : Given $F \in \mathbf{A}^{n}(\mathbf{U})$, its true polyanalytic component $F_{k} \in \mathcal{A}^{k}(\mathbf{U})$ can be recovered by the orthogonal projection of $F$ over the space $\mathcal{A}^{k}(\mathbf{U})$ :

$$
F_{k}(z)=\left\langle F(w), K^{k}(z, w)\right\rangle_{\mathbf{A}^{n}(\mathbf{U})} .
$$

One of the main goals of the present paper is the construction of the unitary transform $B e r^{n}: L^{2}\left(\mathbb{R}^{+}\right) \rightarrow \mathcal{A}^{n-1}(\mathbf{U})$ mentioned above. We will call it the true polyanalytic Bergman transform and define it in terms of the (analytic) Bergman transform

$$
\operatorname{Ber} f(z)=\int_{0}^{\infty} t^{\frac{1}{2}} f(t) e^{i z t} d t
$$

which is unitary Ber $: L^{2}\left(\mathbb{R}^{+}\right) \rightarrow A(\mathbf{U})$. Starting from this, we introduce the true polyanalytic Bergman transform

$$
\operatorname{Ber}^{n} f(z)=\frac{1}{(2 i)^{n} n !}\left(\frac{d}{d z}\right)^{n}\left[s^{n} \operatorname{Ber} f(z)\right] .
$$

It is at this point that the connections to wavelets shows up. Actually, the key in proving the unitarity of $B e r^{n}$ is to recognize that, defining the functions $\Phi_{n}$ as

$$
\mathcal{F} \Phi_{n}(t)=t^{\frac{1}{2}} l_{n}^{0}(2 t),
$$

where $l_{n}^{0}$ is a Laguerre function (see section 2 for definition), the true polyanalytic Bergman transform is related to the wavelet transform by the formula

$$
\operatorname{Ber}^{n} f(z)=s^{-1} W_{\Phi_{n}}\left(\mathcal{F}^{-1} f\right)(x, s) .
$$

The next goal is to define a unitary isomorphism $\mathbf{B e r} r^{n}: L^{2}\left(\mathbb{R}^{+}, \mathbf{C}^{n}\right) \rightarrow \mathbf{A}^{n}(\mathbf{U})$. For $\mathbf{f} \in L^{2}\left(\mathbb{R}^{+}, \mathbf{C}^{n}\right)$, the polyanalytic Bergman transform is

$$
\mathbf{B e} r^{n} \mathbf{f}=\sum_{0 \leq k \leq n-1} B e r^{k} f_{k} .
$$

This provides a unitary operator $\mathbf{B e} r^{n}: L^{2}\left(\mathbb{R}^{+}, \mathbf{C}^{n}\right) \rightarrow \mathbf{A}^{n}(\mathbf{U})$, which relates vector-valued functions to polyanalytic functions. Here vector-valued wavelet transforms enter the picture. We will interpretate the polyanalytic Bergman transform is a special case of a general vector valued wavelet transform defined as

$$
\mathbf{W}_{\mathbf{g}} \mathbf{f}(x, s)=\sum_{0 \leq k \leq n-1} W_{g_{k}} f_{k}(x, s),
$$

where $W_{g_{k}} f_{k}$ stands for the scalar wavelet transform of the kth component of the vector $\mathbf{f}=\left(f_{0}, \ldots, f_{n-1}\right)$, analyzed with the wavelet $g_{k}$, which is a component of the vector-valued analyzing wavelet $\mathbf{g}$. The components of the vector $\mathbf{g}=\left(g_{0}, \ldots, g_{n-1}\right)$ are selected in such a way their Fourier transforms satisfy

$$
\left\langle\mathcal{F} g_{i}, \mathcal{F} g_{j}\right\rangle_{L^{2}\left(\mathbb{R}^{+}, t^{-1}\right)}=\delta_{i, j}
$$


In particular, one can choose the vector-valued analyzing wavelet $\boldsymbol{\Phi}_{n}=\left(\Phi_{0}, \ldots, \Phi_{n-1}\right)$. In doing so, we arrive at the relation between the vector-valued wavelet transform and the polyanalytic Bergman transform:

$$
\mathbf{W}_{\boldsymbol{\Phi}_{n}}\left(\mathcal{F}^{-1} \mathbf{f}\right)(x, s)=s \mathbf{B e r}^{n} \mathbf{f} .
$$

Then, we compute explicitly the reproducing kernels of the spaces $\mathcal{A}^{k}(\mathbf{U})$, required for the orthogonal projections. They are given by the formula

$$
K^{n}(z, w)=\frac{1}{(2 i)^{n} n !}\left(\frac{d}{d z}\right)^{n}\left[s^{n} \Omega_{n}\left(\frac{z+u}{\eta}\right)\right],
$$

where

$$
\Omega_{n}(z)=4(n+z-i)\left(\frac{1}{z+i}\right)^{3}\left(\frac{z-i}{z+i}\right)^{n-1} .
$$

In the final section we study sampling sequences in polyanalytic Bergman spaces. This is is equivalent to the study of wavelet frames with the functions $\Phi_{n}$. The section contains a contribution to the subject of affine density, the study of the density of sequences $\Lambda$ yielding inequalities of the form (wavelet frames):

$$
A\|f\|_{H^{2}(\mathbf{U})}^{2} \leq \sum_{(x, s) \in \Lambda}\left|\left\langle f, T_{x} D_{s} \psi\right\rangle\right|^{2} \leq B\|f\|_{H^{2}(\mathbf{U})}^{2} .
$$

There has been a considerable research activity around the topic of affince density, and currently there are three different aproaches (see [18, [19, [22, [30, [29] and the monograph [23]). The definitions of density in Seip [29] and [18, [19], 22, agree in the case of the hyperbolic lattice $\Lambda(a, b)=\left\{\left(a^{m} b k, a^{m}\right)\right\}$, where the density is $1 / b \log a$, up to constants independent of $a$ and $b$.

It is straightforward to see that there is no universal necessary lower bound for an arbitrary function to generate a frame associated to $\Lambda(a, b)$, in contrast to the situation in Gabor analysis [26, 27. In the literature we have found only one example where such a bound exists. It is the case of the wavelet defined on the Fourier side by $\mathcal{F} \psi_{\alpha}(t)=\mathbf{1}_{[0, \infty]} t^{\alpha} e^{-t}$, where the problem can be translated into a sampling problem in Bergman spaces of analytic functions, which has been completely understood in [29].

Thanks to the connection to polyanalytic Bergman spaces, we will provide a family of examples (which in the case $n=0$ reduces to $\psi_{\alpha}$ ) where an explicit bound on the constant $b \log a$ is shown to be necessary, providing thus the first results in this direction since the sharp conditions associated to the Poisson wavelet. We obtain such conditions by investigating the sampling sequences in the true polyanalytic Bergman space, $\mathcal{A}^{n}(\mathbf{U})$. A sampling sequence in $\mathcal{A}^{n}(\mathbf{U})$ is one originating inequalities of the form

$$
A\|F\|_{\mathcal{A}^{n}(\mathbf{U})}^{2} \leq \sum_{z \in \Gamma(a, b)}|F(z)|^{2} \leq B\|F\|_{\mathcal{A}^{n}(\mathbf{U})}^{2} .
$$

Thanks to the identity

$$
\left\langle f, T_{x} D_{s} \Phi_{n}\right\rangle_{H^{2}(\mathbf{U})}=s \operatorname{Ber}^{n} \mathcal{F} f(z),
$$

this inequality is equivalent to the wavelet frame inequality. To prove the existence of sampling sequences, we use some results from Ascensi and Bruna [3]. Finally, we combine an argument used by Seip [28, with a result from 3 in order to prove a necessary condition for the existence of sampling sequences in $\mathcal{A}^{n}(\mathbf{U})$. In terms 
of wavelet frames, such condiition requires that, if $\left.\mathcal{W}\left(\Phi_{n}, \Lambda\right)\right)$ is a wavelet frame for $H^{2}(\mathbf{U})$, then

$$
b \log a<2 \pi(n+1) .
$$

1.3. Organization of the paper. The outline is as follows. We have a backgound section where we review some known facts concerning wavelets, Laguerre functions and the fundamental facts concerning analytic and polyanalytic Bergman spaces and the connection between Bergman spaces and wavelets provided by the analytic Bergman transform. Then, in the third section, we define a vector-valued version of the continuous wavelet transform and some of its elementary properties. The fourth section is the most important of the paper. We introduce the true polyanalytic and the polyanalytic transforms, and describe their relation with wavelets and vector-valued wavelets. Such a relation is fundamental in order to prove the unitarity of the polyanalitic transforms. In section 6 , the structure of the polyanalytic Bergman spaces is investigated using these new tools. We obtain a sequence of rational fuctions which is orthogonal in the upper half plane, defined by its Rodrigues formula. This sequence of rational functions is a basis of the polyanalytic Bergman space. Then we obtain an explicit formula for the reproducing kernel of the polyanalytic Bergman spaces, in the form of a differential operator which also ressembles a Rodrigues formula. In the last section of the paper we present some results concerning sampling sequences in spaces of polyanalytic functions and their consequences in terms of wavelet frames.

\section{BACKGROUND}

2.1. The wavelet transform. For every $x \in \mathbb{R}$ and $s \in \mathbb{R}^{+}$define the operators translation, modulation and dilation as

$$
T_{x} f(t)=f(t-x) ; \quad M_{x} f(t)=e^{-i x t} f(t),
$$

and

$$
D_{s} f(t)=s^{-\frac{1}{2}} f\left(s^{-1} t\right) .
$$

Fix a function $g \neq 0$. Then the continuous wavelet transform of a function $f$ with respect to a wavelet $g$ is defined, for every $x \in \mathbb{R}, s>0$ as

$$
W_{g} f(x, s)=\left\langle f, T_{x} D_{s} g\right\rangle_{L^{2}(\mathbb{R})} .
$$

The following relations are usually called the orthogonal relations for the wavelet transform. Assume that $g_{1}, g_{2} \in L^{2}(\mathbb{R})$ satisfy

$$
\left\langle\mathcal{F} g_{1}, \mathcal{F} g_{2}\right\rangle_{L^{2}\left(\mathbb{R}^{+}, t^{-1}\right)}<\infty .
$$

Then, for all $f_{1}, f_{2} \in L^{2}(\mathbb{R})$,

$$
\left\langle W_{g_{1}} f_{1}, W_{g_{2}} f_{2}\right\rangle_{L^{2}\left(\mathbf{U}, s^{-2} d x d s\right)}=\left\langle\mathcal{F} g_{1}, \mathcal{F} g_{2}\right\rangle_{L^{2}\left(\mathbb{R}^{+}, t^{-1}\right)}\left\langle f_{1}, f_{2}\right\rangle_{L^{2}(\mathbb{R})} .
$$

A function $g \in L^{2}(\mathbb{R})$ is said to be admissible if

$$
\|\mathcal{F} g\|_{L^{2}\left(\mathbb{R}^{+}, t^{-1}\right)}^{2}=K,
$$

where $K$ is a constant. If $g$ is admissible, then for all $f \in L^{2}(\mathbb{R})$ we have

$$
\left\|W_{g} f\right\|_{L^{2}\left(\mathbf{U}, s^{-2} d x d s\right)}^{2}=K\|f\|_{L^{2}(\mathbb{R})}^{2} .
$$


Therefore, the continuous wavelet transform provides an isometric inclusion, being an isometry when $K=1$.

$$
W_{g}: L^{2}(\mathbb{R}) \rightarrow L^{2}\left(\mathbf{U}, s^{-2} d x d s\right),
$$

If we restrict to functions $f \in H^{2}(\mathbf{U})$, the Hardy space in the upper half plane constituted by analytic functions $f$ such that

$$
\sup _{0<s<\infty} \int_{-\infty}^{\infty}|f(x+i s)|^{2} d x<\infty .
$$

By the Paley-Wiener theorem, $H^{2}(\mathbf{U})$ is constituted by the functions whose Fourier transform,

$$
\mathcal{F} f(\omega)=\int_{\mathbb{R}} f(x) e^{-i x \omega} d x
$$

is supported in $\mathbb{R}^{+}$and belongs to $L^{2}\left(\mathbb{R}^{+}\right)$. Using the action of the Fourier transform on the dilation and translation operators,

$$
\mathcal{F} D_{s} f=D_{\frac{1}{s}} \mathcal{F} f \quad \text { and } \quad \mathcal{F} T_{x} f=M_{-x} \mathcal{F} f,
$$

we can use Plancherel theorem to rewrite the wavelet transform "on the Fourier side" as

$$
W_{g} f(x, s)=\left\langle\mathcal{F} f, M_{-x} D_{\frac{1}{s}} \mathcal{F} g\right\rangle_{L^{2}\left(\mathbb{R}^{+}\right)} .
$$

2.2. The Laguerre functions. The Laguerre polynomials will play a central role in our discussion. One way to define them is by the power series

$$
L_{n}^{\alpha}(x)=\frac{(\alpha+1)_{n}}{n !} \sum_{k=0}^{n} \frac{(-n)_{k}}{(\alpha+1)_{k}} \frac{x^{k}}{k !} .
$$

This is equivalent to the Rodrigues formula

$$
L_{n}^{\alpha}(x)=\frac{e^{x} x^{-\alpha}}{n !} \frac{d^{n}}{d x^{n}}\left[e^{-x} x^{\alpha+n}\right] .
$$

The Laguerre functions are defined as

$$
l_{n}^{\alpha}(x)=\mathbf{1}_{[0, \infty]}(x) e^{-x / 2} x^{\alpha / 2} L_{n}^{\alpha}(x) .
$$

It is well known that, for $\alpha \geq 0$, these functions constitute an orthogonal basis for the space $L^{2}(0, \infty)$.

\section{BERGMAN SPACES}

3.1. Analytic and polyanalytic Bergman spaces. With the Wirtinger diferential operator notation,

$$
\frac{d}{d z}=\frac{1}{2}\left(\frac{\partial}{\partial x}-i \frac{\partial}{\partial s}\right), \quad \frac{d}{d \bar{z}}=\frac{1}{2}\left(\frac{\partial}{\partial x}+i \frac{\partial}{\partial s}\right),
$$

a complex valued function $f$, is said to be analytic in a domain, if, for every $z$ in such domain, it satisfies

$$
\frac{d}{d \bar{z}} f(z)=0
$$

More generally, $f$ is said to be polyanalytic of order $n$ if

$$
\left(\frac{d}{d \bar{z}}\right)^{n} f(z)=0
$$


Then $A(\mathbf{U})$ stands for the Bergman space in the upper half plane, constituted by the analytic functions in $\mathbf{U}$ such that

$$
\int_{\mathbf{U}}|f(z)|^{2} d x d s<\infty .
$$

The space constituted by the polyanalytic functions of order $n$, equipped with the same norm as the Bergman space is called the polyanalytic Bergman space, $\mathbf{A}^{n}(\mathbf{U})$. With this notation, $\mathbf{A}^{1}(\mathbf{U})=A(\mathbf{U})$. Consider also the true polyanalytic Bergman space, $\mathcal{A}^{n}(\mathbf{U})$, defined as

$$
\mathcal{A}^{n-1}(\mathbf{U})=\mathbf{A}^{n}(\mathbf{U}) \ominus \mathbf{A}^{n-1}(\mathbf{U}),
$$

so that the following decomposition holds:

$$
\mathbf{A}^{n}(\mathbf{U})=\mathcal{A}^{0}(\mathbf{U}) \oplus \ldots \oplus \mathcal{A}^{n-1}(\mathbf{U}) .
$$

3.2. The Bergman transform. We can relate the wavelet transform to Bergman spaces of analytic functions, by choosing the window $\psi_{\alpha}$ such that

$$
\mathcal{F} \psi_{\alpha}(t)=\mathbf{1}_{[0, \infty]} t^{\alpha} e^{-t} .
$$

Writing $z=x+$ si gives

$$
\mathcal{F} T_{-x} D_{s} \psi_{\alpha}(t)=\mathbf{1}_{[0, \infty]} s^{\alpha+\frac{1}{2}} t^{\alpha} e^{i z t}
$$

and

$$
W_{\overline{\psi_{\alpha}}} f(-x, s)=s^{\alpha+\frac{1}{2}} \int_{0}^{\infty} t^{\alpha}(\mathcal{F} f)(t) e^{i z t} d t .
$$

Considering $f \in H^{2}(\mathbf{U})$, then $\mathcal{F} f \in L^{2}\left(\mathbb{R}^{+}\right)$. This motivates the definition of the Bergman transform of order $\alpha$ as the analytic part of (3.5):

$$
\operatorname{Ber}_{\alpha} f(z)=s^{-\alpha} W_{\overline{\psi_{\alpha-\frac{1}{2}}}}\left(\mathcal{F}^{-1} f\right)(-x, s)=\int_{0}^{\infty} t^{\alpha-\frac{1}{2}} f(t) e^{i z t} d t .
$$

We will write

$$
\operatorname{Ber} f(z)=\operatorname{Ber}_{1} f(z)=\int_{0}^{\infty} t^{\frac{1}{2}} f(t) e^{i z t} d t
$$

in order to obtain an isometric transformation

$$
\operatorname{Ber}: L^{2}\left(\mathbb{R}^{+}\right) \rightarrow A(\mathbf{U}) .
$$

\section{A Continuous Vector VAlued WAVElet transform}

Now, consider the Hilbert space $\mathcal{H}=L^{2}\left(\mathbb{R}, \mathbf{C}^{n}\right)$ consisting of vector-valued functions $\mathbf{f}=\left(f_{0}, \ldots, f_{n-1}\right)$ with the inner product

$$
\langle\mathbf{f}, \mathbf{g}\rangle_{\mathcal{H}}=\sum_{0 \leq k \leq n-1}\left\langle f_{k}, g_{k}\right\rangle_{L^{2}(\mathbb{R})} .
$$

Definition 1. Let $\mathbf{g}=\left(g_{0}, \ldots, g_{n-1}\right)$ be a vector of functions in $\mathcal{H}$ such that

$$
\left\langle\mathcal{F} g_{i}, \mathcal{F} g_{j}\right\rangle_{L^{2}\left(\mathbb{R}^{+}, t^{-1}\right)}=\delta_{i, j}
$$

The continuous vector valued wavelet transform of a function $\mathbf{f}=\left(f_{1}, \ldots, f_{n-1}\right)$ with respect to the vectorial window $\mathbf{g}$ is defined, for every $x \in \mathbb{R}, s \in \mathbb{R}^{+}$, as

$$
\mathbf{W}_{\mathbf{g}} \mathbf{f}(x, \omega)=\left\langle\mathbf{f}, D_{\mathbf{s}} T_{x} \mathbf{g}\right\rangle_{\mathcal{H}} .
$$


We can also write

$$
\mathbf{W}_{\mathbf{g}} \mathbf{f}(x, s)=\sum_{0 \leq k \leq n-1} W_{g_{k}} f_{k}(x, s)
$$

This defines a map

$$
\mathbf{W}_{\mathbf{g}} \mathbf{f}: \mathcal{H} \rightarrow L^{2}\left(\mathbf{U}, s^{-2} d s d s\right) .
$$

The orthogonality condition imposed on the vector $\mathbf{g}$ allows the superwavelet transform to retain most of the properties of the scalar Wavelet transform. In particular, we have vector valued versions of the isometric property and orthogonality relations.

Proposition 1. Let $\mathbf{g}$ satisfy [4.2). Then, for $\mathbf{f}_{1}, \mathbf{f}_{2}, \in \mathcal{H}$,

$$
\left\langle\mathbf{W}_{\mathbf{g}} \mathbf{f}_{1}, \mathbf{W}_{\mathbf{g}} \mathbf{f}_{2}\right\rangle_{L^{2}\left(\mathbf{U}, s^{-2} d z\right)}=\left\langle\mathbf{f}_{1}, \mathbf{f}_{2}\right\rangle_{\mathcal{H}} \text {. }
$$

In particular, $\mathbf{W}_{\mathbf{g}} \mathbf{f}$ is an isometry between Hilbert spaces, that is

$$
\left\|\mathbf{W}_{\mathbf{g}} \mathbf{f}\right\|_{L^{2}\left(\mathbf{U}, s^{-2} d z\right)}=\|\mathbf{f}\|_{\mathcal{H}} .
$$

Proof. From (2.2) and (4.2),

$$
\left\langle W_{g_{k}} f_{k}, W_{g_{j}} f_{j}\right\rangle_{L^{2}\left(\mathbf{U}, s^{-2} d x d s\right)}=\left\langle f_{k}, f_{j}\right\rangle_{L^{2}(\mathbb{R})} \times \delta_{k, j} .
$$

Then,

$$
\begin{aligned}
\left\langle\mathbf{W}_{\mathbf{g}} \mathbf{f}_{1}, \mathbf{W}_{\mathbf{g}} \mathbf{f}_{2}\right\rangle_{L^{2}\left(\mathbf{U}, s^{-2} d x d s\right)} & =\sum_{0 \leq k, j \leq n-1}\left\langle W_{g_{k}} f_{1, k}, W_{g_{j}} f_{2, k}\right\rangle_{L^{2}\left(\mathbf{U}, s^{-2} d x d s\right)} \\
& =\sum_{0 \leq k, j \leq n-1}\left\langle f_{1, k}, f_{2, j}\right\rangle_{L^{2}\left(\mathbf{U}, s^{-2} d x d s\right)} \times \delta_{k, j} \\
& =\sum_{0 \leq k \leq n-1}\left\langle f_{1, k}, f_{2, k}\right\rangle_{L^{2}\left(\mathbf{U}, s^{-2} d x d s\right)} \\
& =\left\langle\mathbf{f}_{1}, \mathbf{f}_{2}\right\rangle_{\mathcal{H}} .
\end{aligned}
$$

Now let $\mathbf{W}_{\mathbf{g}}$ stand for the subspace of $L^{2}\left(\mathbb{R}^{2}\right)$ constituted by the image of $\mathcal{H}$ under the vector valued wavelet transform $\mathbf{W}_{\mathbf{g}} \mathbf{f}$ :

$$
\mathbf{W}_{\mathbf{g}}=\left\{\mathbf{W}_{\mathbf{g}} \mathbf{f}: \mathbf{f} \in \mathcal{H}\right\} .
$$

Since

$$
\mathbf{W}_{\mathbf{g}} \mathbf{f}=\sum_{0 \leq k \leq n-1} W_{g_{k}} f_{k}
$$

and

$$
\left\langle W_{g_{k}} f_{k}, W_{g_{j}} f_{j}\right\rangle_{L^{2}\left(\mathbf{U}, s^{-2} d x d s\right)}=\delta_{k, j},
$$

we know that every $F \in \mathbf{W}_{g}$ can be written in a unique way in the form

$$
F=F_{0}+\ldots+F_{n-1} .
$$

As a result,

$$
\mathbf{W}_{\mathbf{g}}=\mathcal{W}_{g_{0}} \oplus \ldots \oplus \mathcal{W}_{g_{n-1}},
$$

where

$$
\mathcal{W}_{g_{j}}=\left\{W_{g_{j}} f: f \in L^{2}(\mathbb{R})\right\} .
$$


Proposition 2. The space $\mathbf{W}_{\mathbf{g}}$ is a Hilbert space with reproducing kernel given by

$$
\mathbf{k}(z, w)=\left\langle T_{\eta} D_{u} \mathbf{g}, T_{x} D_{s} \mathbf{g}\right\rangle_{\mathcal{H}}=\sum_{j=0}^{n-1} k_{j}(z, w)
$$

where $k_{j}(z, w)$ is the reproducing kernel of $\mathcal{W}_{g_{j}}$.

Proof. Let $\mathbf{F} \in \mathbf{W}_{\mathbf{g}}$. There exists $\mathbf{f} \in \mathcal{H}$ such that $\mathbf{F}=\mathbf{W}_{\mathbf{g}} \mathbf{f}$. By definition, $\mathbf{k}(z,)=.\mathbf{W}_{g}\left(T_{x} D_{\mathbf{s}} \mathbf{g}\right)$. Thus, using (4.4),

$$
\begin{aligned}
\langle\mathbf{F}, \mathbf{k}(z, .)\rangle_{L^{2}\left(\mathbf{U}, s^{-2} d z\right)} & =\left\langle\mathbf{W}_{\mathbf{g}} \mathbf{f}, \mathbf{W}_{g}\left(T_{x} D_{s} \mathbf{g}\right)\right\rangle_{L^{2}\left(\mathbf{U}, s^{-2} d z\right)} \\
& =\left\langle\mathbf{f}, T_{x} D_{s} \mathbf{g}\right\rangle_{\mathcal{H}} \\
& =\mathbf{F}(z) .
\end{aligned}
$$

The second inequality follows from the well known fact that the reproducing kernel of the space $\mathcal{W}_{g_{j}}$ is given by $\left\langle T_{\eta} D_{u} g_{j}, T_{x} D_{s} g_{j}\right\rangle_{L^{2}(\mathbb{R})}$.

Through the paper, we will restrict ourselfs to vectors $\mathbf{f}$ such that the Fourier transform of each of its components is supported in $\mathbb{R}^{+}$and belongs to $L^{2}\left(\mathbb{R}^{+}\right)$. In such a case, $\mathcal{H}=H^{2}\left(\mathbf{U}, \mathbf{C}^{n}\right)$ and, on the Fourier side, the notation $\mathcal{H}^{+}=$ $L^{2}\left(\mathbb{R}^{+}, \mathbf{C}^{n}\right)$, will be used.

\section{The Polyanalytic Bergman transform}

In this section we will study a special case of the continuous vector valued wavelet transform, when the vector is defined in terms of Laguerre functions. First we treat the scalar case, which originates a unitary map onto the true polyanalytic Bergman space. We show that the required unitary mappings can be related to special wavelet transforms and, via a connection to the previous section, we define the vector valued polyanalytic transform onto the polyanalytic space (which we call the polyanalytic Bergman transform).

5.1. The true polyanalytic Bergman transform. First we will study the transform that allows, in the multiplexing context explained in the introduction, to send each signal $f_{k} \in L^{2}\left(\mathbb{R}^{+}\right)$to a space $\mathcal{A}^{k}(\mathbf{U})$.

Definition 2. The true polyanalytic Bergman transform of order $n$ is the transform mapping every $f \in L^{2}\left(\mathbb{R}^{+}\right)$to

$$
\operatorname{Ber}^{n} f(z)=\frac{1}{(2 i)^{n} n !}\left(\frac{d}{d z}\right)^{n}\left[s^{n} F(z)\right],
$$

where $F=\operatorname{Ber} f$ and $f \in L^{2}\left(\mathbb{R}^{+}\right)$.

The purpose of this section is to prove that the transform $B e r^{n}$ is unitary

$$
\operatorname{Ber}^{n}: L^{2}\left(\mathbb{R}^{+}\right) \rightarrow \mathcal{A}^{n}(\mathbf{U}) .
$$

We will need some identities which have independent interest. First observe that, since

we have

$$
L_{n}^{0}(t)=\sum_{k=0}^{n}(-1)^{k}\left(\begin{array}{l}
n \\
k
\end{array}\right) \frac{t^{k}}{k !}
$$

$$
l_{n}^{0}(t)=\sum_{k=0}^{n}(-1)^{k}\left(\begin{array}{l}
n \\
k
\end{array}\right) \frac{1}{k !} \mathbf{1}_{[0, \infty]}(x) t^{k} e^{-t / 2}=t^{-\frac{1}{2}} \sum_{k=0}^{n}(-1)^{k}\left(\begin{array}{l}
n \\
k
\end{array}\right) \mathcal{F} \psi_{k+\frac{1}{2}}\left(\frac{t}{2}\right) .
$$


Due to this observation, it is reasonable to expect that the functions $\Phi_{n}$, defined by

$$
\mathcal{F} \Phi_{n}(t)=t^{\frac{1}{2}} l_{n}^{0}(2 t)
$$

will play a distinguished role in our analysis. Indeed, an essential step in the proof of the unitary property is to write (5.1) in terms of a wavelet transform with analysing wavelet $\Phi_{n}$.

Proposition 3. The true polyanalytic Bergman transform of order $n$ can be written as:

(1) A polyanalytic function of order $n+1$ :

$$
\operatorname{Ber}^{n} f(z)=\frac{1}{(2 i)^{n} n !} \sum_{k=0}^{n}(2 i)^{k}\left(\begin{array}{l}
n \\
k
\end{array}\right) \frac{1}{k !} s^{k} F^{(k)}(z) .
$$

(2) In terms of analytic Bergman transforms of different orders:

$$
\operatorname{Ber}^{n} f(z)=\sum_{k=0}^{n}(-2)^{k}\left(\begin{array}{l}
n \\
k
\end{array}\right) s^{k} \operatorname{Ber}_{k+1} f(z) .
$$

(3) In terms of a wavelet transform:

$$
\operatorname{Ber}^{n} f(z)=s^{-1} W_{\Phi_{n}}\left(\mathcal{F}^{-1} f\right)(x, s) .
$$

Proof. The first identity follows from a standard application of Leibnitz formula. Then, since

we have

$$
\frac{d}{d \bar{z}} s^{k}=\frac{1}{2} k s^{k-1}
$$

$$
\left(\frac{d}{d \bar{z}}\right)^{n+1} \operatorname{Ber}^{n} f(z)=0
$$

and $\operatorname{Ber}^{n} f$ is polyanalytic of order $n+1$. To prove (5.5), observe that diferentiating (3.7) under the integral sign gives

$$
F^{(k)}(z)=\left(\frac{d}{d z}\right)^{k} \operatorname{Ber} f(z)=i^{k} \operatorname{Ber}_{k+1} f(z) .
$$

Applying this to (5.4) gives (5.5).

Now (5.6). Combining (5.2) with (5.3) and inverting the Fourier transform gives

$$
\Phi_{n}(t)=\sum_{k=0}^{n}(-2)^{k}\left(\begin{array}{l}
n \\
k
\end{array}\right) \psi_{k+\frac{1}{2}}(t) .
$$

Then,

$$
\begin{aligned}
W_{\Phi_{n}} f(x, s) & =\sum_{k=0}^{n}(-2)^{k}\left(\begin{array}{l}
n \\
k
\end{array}\right) W_{\overline{\psi_{k+\frac{1}{2}}}} f(-x, s) \\
& =\sum_{k=0}^{n}(-2)^{k}\left(\begin{array}{l}
n \\
k
\end{array}\right) s^{k+1} \operatorname{Ber}_{k+1} \mathcal{F} f(z) \\
& =s \operatorname{Ber}^{n}(\mathcal{F} f)(z) .
\end{aligned}
$$

and (5.6) follows. 
Now we can prove the main result. The idea consists in writing the wavelet transform (5.6) as a composition of several unitary operators and is suggested by the techniques used in [31] and 21]. We need to introduce two auxiliary operators. For convenience write $L^{2}(\mathbf{U})=L^{2}(\mathbb{R}) \otimes L^{2}\left(\mathbb{R}^{+}\right)$and define the unitary operators $U_{1,2}: L^{2}(\mathbb{R}) \otimes L^{2}\left(\mathbb{R}^{+}\right) \rightarrow L^{2}(\mathbb{R}) \otimes L^{2}\left(\mathbb{R}^{+}\right):$

$$
\begin{aligned}
& U_{1}(F)(x, s)=\left(\mathcal{F}^{-1} \otimes I\right)(F)(x, s) \\
& U_{2}(F)(x, s)=\frac{1}{\sqrt{2|x|}} F\left(x, \frac{s}{2|x|}\right) .
\end{aligned}
$$

We will need the following result of Vasilevski 31.

Theorem A [31, Corollary 4.2] Let $L_{n}$ stand for the space generated by $1_{[0, \infty]} l_{n}^{0}$ The operator $U=U_{2} U_{1}$,

$$
U: \mathcal{A}^{n}(\mathbf{U}) \rightarrow L^{2}\left(\mathbb{R}^{+}\right) \otimes L_{n}
$$

such that, given $f \in \mathcal{A}^{n}(\mathbf{U})$,

$$
(U f)(x, s)=\mathbf{1}_{[0, \infty]}(x) f(x) l_{n}^{0}(s),
$$

is unitary.

We now combine Theorem A with Proposition 3 to prove the main result.

Theorem 1. The transform $\operatorname{Ber}^{n}: L^{2}\left(\mathbb{R}^{+}\right) \rightarrow \mathcal{A}^{n}(\mathbf{U})$ is unitary.

Proof. Consider the unitary operator

$$
R_{k}: L^{2}\left(\mathbb{R}^{+}\right) \rightarrow L^{2}\left(\mathbb{R}^{+}\right) \otimes L_{k}
$$

defined by

$$
\left(R_{k} f\right)(x, s)=\mathbf{1}_{[0, \infty]}(x) f(x) l_{k}^{0}(s) .
$$

Then the composition $U^{-1} R_{k}$ is also unitary

$$
U^{-1} R_{k}: L^{2}\left(\mathbb{R}^{+}\right) \rightarrow \mathcal{A}^{k}(\mathbf{U})
$$

We will now show that this transform is exactly $B e r^{n}$. From the definition of $U_{2}$ it is easy to see that

$$
U_{2}^{-1}(F)(x, s)=\sqrt{2|x|} F(x, 2|x| s)
$$

and

$$
\left(U_{2}^{-1} R_{k} f\right)(x, s)=\mathbf{1}_{[0, \infty]}(x) \sqrt{2 x} f(x) l_{k}^{0}(2 x s)
$$

Applying $U_{1}^{-1}$ gives

$$
\begin{aligned}
\left(U^{-1} R_{k} f\right)(x, s) & =s^{-1} \int_{0}^{\infty} f(t) s^{\frac{1}{2}}(2 t s)^{\frac{1}{2}} l_{k}^{0}(2 t s) e^{i x t} d t \\
& =s^{-1} \int_{\mathbb{R}}\left(\mathcal{F}^{-1} f\right)(t) s^{-\frac{1}{2}} \overline{\Phi_{n}\left(s^{-1}(t-x)\right)} d t \\
& =s^{-1} W_{\Phi_{n}}\left(\mathcal{F}^{-1} f\right)(x, s) \\
& =\operatorname{Ber}^{n} f(z),
\end{aligned}
$$

by using the identity (5.6). 
5.2. The polyanalytic Bergman transform. Now, consider the Hilbert space $\mathcal{H}^{+}=L^{2}\left(\mathbb{R}^{+}, \mathbf{C}^{n}\right)$ consisting of vector-valued functions $\mathbf{f}=\left(f_{0}, \ldots, f_{n-1}\right)$ with the inner product

$$
\langle\mathbf{f}, \mathbf{g}\rangle_{\mathcal{H}^{+}}=\sum_{0 \leq k \leq n-1}\left\langle f_{k}, g_{k}\right\rangle_{L^{2}\left(\mathbb{R}^{+}\right)} .
$$

Definition 3. The polyanalytic Bergman transform of order $\mathrm{n}$ is defined, for $\mathbf{f} \in$ $\mathcal{H}^{+}$as

$$
\operatorname{Ber} r^{n} \mathbf{f}=\sum_{0 \leq k \leq n-1} B e r^{k} f_{k} .
$$

If we take, then we have the following relation with the polyanalytic Bergman transform:

Theorem 2. The polyanalytic Bergman transform of order $\mathrm{n}$ is a unitary operator

$$
\operatorname{Be} r^{n}: \mathcal{H}^{+} \rightarrow \mathbf{A}^{n}(\mathbf{U})
$$

Proof. To see that it is onto, let $\mathbf{F} \in \mathbf{A}^{n}(\mathbf{U})$. Then, using (3.2), write

$$
\mathbf{F}=F_{0}+\ldots+F_{n-1},
$$

with $F_{k} \in A^{k}(\mathbf{U}), k=0, \ldots, n-1$. Since $B e r^{k}$ is onto, for every $k=0, \ldots, n-1$ there exists $f_{k} \in L^{2}\left(\mathbb{R}^{+}\right)$such that $F_{k}=B e r^{k} f_{k}$. To prove the isometry, we first relate the polyanalytic Bergman transform to the vector valued wavelet transform with the vectorial window $\boldsymbol{\Phi}_{n}=\left(\Phi_{0}, \ldots, \Phi_{n-1}\right)$, using the identity $\operatorname{Ber}^{n} f(z)=$ $s^{-1} W_{\Phi_{n}}\left(\mathcal{F}^{-1} f\right)(x, s)$ :

$$
\mathbf{W}_{\mathbf{\Phi}_{n}}\left(\mathcal{F}^{-1} \mathbf{f}\right)(x, s)=\sum_{0 \leq k \leq n-1} W_{\mathbf{\Phi}_{k}}\left(\mathcal{F}^{-1} f_{k}\right)(x, s)=\sum_{0 \leq k \leq n-1} s B e r^{k} f_{k}=s \mathbf{B e r}^{n} \mathbf{f} .
$$

Now, combining this with 4.5.,

$$
\left\|\operatorname{Ber}^{n} \mathbf{f}\right\|_{\mathbf{A}^{n}(\mathbf{U})}=\left\|\mathbf{W}_{\mathbf{\Phi}_{n}}\left(\mathcal{F}^{-1} \mathbf{f}\right)\right\|_{\mathbf{L}^{2}\left(\mathbf{U}_{, s^{-2}} d x d s\right)}=\left\|\mathcal{F}^{-1} \mathbf{f}\right\|_{\mathcal{H}}=\|\mathbf{f}\|_{\mathcal{H}^{+}} .
$$

\section{The structure of polyanalytic Bergman spaces}

The purpose of this section is to apply the connection to wavelet transforms to study polyanalytic Bergman spaces. We will obtain an orthogonal basis for $\mathbf{A}^{n}(\mathbf{U})$ and compute an explicit formula for the reproducing kernel. The reproducing kernel, $K^{n}(z, w)$, of the true polyanalytic Bergman space $\mathcal{A}^{n}(\mathbf{U})$ is also very important, since once we have a function $F \in \mathbf{A}^{n}(\mathbf{U})$, we can recover its true polyanalytic component $F_{k} \in \mathcal{A}^{k}(\mathbf{U})$ by the orthogonal projecion over the space $\mathcal{A}^{k}(\mathbf{U})$, which is given by the formula

$$
F_{k}(z)=\left\langle F(w), K^{k}(z, w)\right\rangle_{\mathbf{A}^{n}(\mathbf{U})} .
$$

Our formulas will be given in a form of differential operators which are reminiscent of the Rodrigues formula, a well known structure formula in the theory of classic orthogonal polynomials. 
6.1. An orthogonal basis. Consider the functions $\Psi_{n}^{\beta}$, for every $n \geq 0$ and $\beta>1$ :

$$
\Psi_{n}^{\beta}(z)=(2 i)^{\beta+1} \frac{\Gamma(\beta+n)}{n !}\left(\frac{z-i}{z+i}\right)^{n}\left(\frac{1}{z+i}\right)^{\beta} .
$$

It is well known that these functions constitute a basis of $A_{\beta-2}(\mathbf{U})$. A calculation using the special function formula

$$
\int_{0}^{\infty} x^{\alpha} L_{n}^{\alpha}(x) e^{-x s} d x=\frac{\Gamma(\alpha+n+1)}{n !} s^{-\alpha-n-1}(s-1)^{n}
$$

gives

$$
\operatorname{Ber}_{\alpha} l_{n}^{2 \alpha-1}=\Psi_{n}^{2 \alpha}
$$

Now write

$$
\Psi_{n}(z)=\Psi_{n}^{2}(z)=(2 i)^{3} \frac{\Gamma(2+n)}{n !}\left(\frac{z-i}{z+i}\right)^{n}\left(\frac{1}{z+i}\right)^{2}
$$

to denote a basis of $A(\mathbf{U})$, so that

$$
\operatorname{Ber} l_{n}^{1}=\Psi_{n} .
$$

Definition 4. Define a set of functions by

$$
e_{n, m}(z)=\frac{1}{(2 i)^{n} n !}\left(\frac{d}{d z}\right)^{n}\left[s^{n} \Psi_{m}(z)\right] .
$$

Proposition 4. The set $\left\{e_{k, m}\right\}_{k \geq 0,0 \leq m<n}$ is an orthonormal basis of $\mathbf{A}^{n}(\mathbf{U})$.

Proof. Since

$$
e_{n, k}(z)=\operatorname{Ber}^{n} l_{k}^{1}=s^{-1} W_{\Phi_{n}}\left(\mathcal{F}^{-1} l_{k}^{1}\right)(x, s),
$$

the orthogonality follows from (2.2):

$$
\begin{aligned}
\left\langle e_{n, k}, e_{l, j}\right\rangle_{L^{2}(\mathbf{U}, d z)} & =\left\langle W_{\Phi_{n}}\left(\mathcal{F}^{-1} l_{k}^{1}\right), W_{\Phi_{l}}\left(\mathcal{F}^{-1} l_{j}^{1}\right)\right\rangle_{L^{2}\left(\mathbf{U}, s^{-2} d z\right)} \\
& =\left\langle l_{n}^{0}, l_{l}^{0}\right\rangle_{L^{2}\left(\mathbb{R}^{+}\right)}\left\langle\mathcal{F}^{-1} l_{k}^{1}, \mathcal{F}^{-1} l_{j}^{1}\right\rangle_{H^{2}(\mathbf{U})} \\
& =\delta_{n, l} \delta_{k, j} .
\end{aligned}
$$

The unitarity of $B e r^{n}$ shows that, for every $m,\left\{e_{k, m}\right\}_{k \geq 0}$ spans $\mathcal{A}^{m}(\mathbf{U})$, since $\left\{l_{n}^{1}\right\}_{n \geq 0}$ spans $L^{2}\left(\mathbb{R}^{+}\right)$. From the decomposition (3.2), every element in $\mathbf{A}^{n}(\mathbf{U})$ can be written as a linear combination of elements of $\left\{\mathcal{A}^{m}(\mathbf{U})\right\}_{m<n}$. Therefore $\left\{e_{k, m}\right\}_{k \geq 0,0 \leq m<n}$ spans $\mathbf{A}^{n}(\mathbf{U})$.

Corollary 1. The set $\left\{e_{k, m}\right\}_{, 0 \leq m<n}$ is an orthonormal basis of $\mathcal{A}^{k}(\mathbf{U})$.

Proof. This follows immediately from the decomposition (3.2).

6.2. The reproducing kernel. In our computations of the reproducing kernels we will need the following relation, which says essentially that the Bergman transform intertwines the representation of the affine group in $L^{2}\left(\mathbb{R}^{+}\right)$with its representation in the Bergman space:

$$
\operatorname{Ber}\left(M_{-\mu} D_{\frac{1}{\eta}} f\right)=s^{-1} \operatorname{Ber}(f)\left(\frac{z+u}{\eta}\right) ;
$$

The identity (6.1) follows from the change of variables:

$$
\int_{0}^{\infty} t^{\frac{1}{2}} e^{i z t}\left(M_{-\mu} D_{\frac{1}{\eta}} f\right)(t) d t=\int_{0}^{\infty} t^{\frac{1}{2}} e^{i\left(\frac{z+u}{\eta}\right) t} f(t) d t
$$


It is interesting to observe that, defining a transform by $T=\operatorname{Ber}(\mathcal{F} f)$ and $M f(t)=$ $t^{\frac{1}{2}} f(t)$, we have the following commutative diagram:

$$
\begin{array}{clcc}
H^{2}(\mathbf{U}) & T & A^{2}(\mathbf{U}) \\
\mathcal{F} \downarrow & & \downarrow \mathcal{F} \\
L^{2}\left(\mathbb{R}^{+}\right) & M & L^{2}\left(\mathbb{R}^{+}, t^{-1} d t\right)
\end{array}
$$

The Fourier isometry on the right column is the Paley-Wiener theorem for the Bergman space 9 .

Now we compute the reproducing kernel of the wavelet space $\mathcal{W}_{\Phi_{n}}$.

Theorem 3. The reproducing kernel of $\mathcal{W}_{\Phi_{n}}$ is given by

$$
k^{n}(z, w)=\frac{1}{n !(2 i)^{n}} \frac{s}{\eta}\left(\frac{d}{d z}\right)^{n}\left[s^{n} \Omega_{n}\left(\frac{z+u}{\eta}\right)\right]
$$

where

$$
\Omega_{n}(z)=4(n+z-i)\left(\frac{1}{z+i}\right)^{3}\left(\frac{z-i}{z+i}\right)^{n-1} .
$$

Proof. The reproducing kernel of $\mathcal{W}_{\Phi_{n}}$ is

$$
\begin{aligned}
k^{n}(z, w) & =\left\langle T_{-\mu} D_{\eta} \Phi_{n}, T_{-x} D_{s} \Phi_{n}\right\rangle_{H^{2}(\mathbf{U})} \\
& =s \operatorname{Ber}^{n}\left(M_{-\mu} D_{\frac{1}{\eta}} \mathcal{F} \Phi_{n}\right)(z) \\
& =\frac{s}{n !(2 i)^{n}} \sum_{k=0}^{n}(2 i)^{k}\left(\begin{array}{l}
n \\
k
\end{array}\right) \frac{s^{k}}{k !}\left(\frac{d}{d z}\right)^{k}\left[\operatorname{Ber}\left(M_{-\mu} D_{\frac{1}{\eta}} \mathcal{F} \Phi_{n}\right)(z)\right] \\
& =\frac{s}{n !(2 i)^{n}}\left(\frac{d}{d z}\right)^{n}\left[s^{n} \operatorname{Ber}\left(M_{-\mu} D_{\frac{1}{\eta}} \mathcal{F} \Phi_{n}\right)(z)\right]
\end{aligned}
$$

Now, (6.1) gives

$$
k^{n}(z, w)=\frac{1}{(2 i)^{n} n !} \frac{s}{\eta}\left(\frac{d}{d z}\right)^{n}\left[s^{n} \operatorname{Ber}\left(\mathcal{F} \Phi_{n}\right)\left(\frac{z+u}{\eta}\right)\right] .
$$

We just need to compute

$$
\begin{aligned}
\operatorname{Ber}\left(\mathcal{F} \Phi_{n}\right) & =\int_{0}^{\infty} t l_{n}^{0}(2 t) e^{i t z} d t=\frac{1}{i} \frac{d}{d z} \int_{0}^{\infty} l_{n}^{0}(2 t) e^{i t z} d t \\
& =\frac{4}{i} \frac{d}{d z}\left[\left(\frac{z-i}{z+i}\right)^{n} \frac{1}{z+i}\right]=\Omega_{n}(z),
\end{aligned}
$$

and the formula is proved.

The next Lemma can be used to transfer properties from the spaces $\mathcal{W}_{\Phi_{n}}$ to the spaces $\mathcal{A}^{n}(\mathbf{U})$.

Lemma 1. The operator

is unitary.

$$
\begin{gathered}
E: \mathcal{W}_{\Phi_{n}} \rightarrow \mathcal{A}^{n}(\mathbf{U}) \\
\quad f \rightarrow s^{-1} f(x, s),
\end{gathered}
$$

Proof. Clearly, $E$ is isometric. Since $l_{m}^{1}$ is a basis of $L^{2}\left(\mathbb{R}^{+}\right)$, then $W_{\Phi_{n}}\left(\mathcal{F}^{-1} l_{m}^{1}\right)$ is a basis of $\mathcal{W}_{\Phi_{n}}$. Then

$$
E\left(W_{\Phi_{n}}\left(\mathcal{F}^{-1} l_{m}^{1}\right)\right)=s^{-1} W_{\Phi_{n}}\left(\mathcal{F}^{-1} l_{m}^{1}\right)(x, s)=\operatorname{Ber}^{n} l_{m}^{1}(z)=e_{n, m}(z) .
$$

Thus, $E\left(\mathcal{W}_{\Phi_{n}}\right)$ is dense in $\mathcal{A}^{n}(\mathbf{U})$. 
Theorem 4. The reproducing kernels of the spaces $\mathcal{A}^{n}(\mathbf{U}), K^{n}(z, w)$, are given by

$$
K^{n}(z, w)=\frac{1}{n !(2 i)^{n}}\left(\frac{d}{d z}\right)^{n}\left[s^{n} \Omega_{n}\left(\frac{z+u}{\eta}\right)\right],
$$

The reproducing kernels of the spaces, $\mathbf{A}^{n}(\mathbf{U}), \mathbf{K}^{n}(z, w)$, are given by

$$
\mathbf{K}^{n}(z, w)=\sum_{k=0}^{n-1} \frac{1}{n !(2 i)^{n}}\left(\frac{d}{d z}\right)^{n}\left[s^{n} \Omega_{n}\left(\frac{z+u}{\eta}\right)\right] .
$$

Proof. Let $f \in \mathcal{A}^{n}(\mathbf{U})$. Then, by the above Lemma, $s f(z) \in \mathcal{W}_{\Phi_{n}}$. Therefore,

$$
s f(z)=\left\langle k^{n}(z, w), \eta f(w)\right\rangle_{\mathcal{W}_{\Phi_{n}}},
$$

and

$$
f(z)=\left\langle\frac{\eta}{s} k^{n}(z, w), f(w)\right\rangle_{\mathbf{A}^{n}(\mathbf{U})} .
$$

We conclude that $K^{n}(z, w)=\frac{\eta}{s} k^{n}(z, w)$. The second assertion follows imediately from (4.9).

\section{SAMPLing SEQUENCES AND WAVELET FRAMES}

This section is devoted to sampling and frames for Wavelet frames and superframes. There are several approaches to general sampling and stability problems (see, for instance [11] and [12]), but we will follow mainly the one in [3], where, fixed an analyzing wavelet, the space of all continuous transforms (the "model space") is considered, in order to translate the frame problem in a sampling problem for such a model space.

Now, we will denote by $\Gamma(a, b)$ the set $\left\{z_{m k}=a^{m}(b k+i)\right\}$. We say that $\Gamma$ is a sampling sequence for $\mathcal{A}^{n}(\mathbf{U})$ if there exist $A, B>0$ such that, for every $F \in \mathcal{A}^{n}(\mathbf{U})$,

$$
A\|F\|_{\mathcal{A}^{n}(\mathbf{U})}^{2} \leq \sum_{z \in \Gamma(a, b)} s^{2}|F(z)|^{2} \leq B\|F\|_{\mathcal{A}^{n}(\mathbf{U})}^{2} .
$$

We say that $\mathcal{W}(\psi, \Gamma(a, b)))$ is a wavelet frame for $H^{2}(\mathbf{U})$ if

$$
A\|f\|_{H^{2}(\mathbf{U})}^{2} \leq \sum_{j, k}\left|\left\langle f, T_{a^{j} b k} D_{a^{j}} \psi\right\rangle\right|^{2} \leq B\|f\|_{H^{2}(\mathbf{U})}^{2} .
$$

Since

$$
\left\langle f, T_{x} D_{s} \Phi_{n}\right\rangle_{H^{2}(\mathbf{U})}=W_{\Phi_{n}} f(x, s)=s \operatorname{Ber}^{n} \mathcal{F} f(z),
$$

it is plain that $\Gamma$ is a sampling sequence for $\mathcal{A}^{n}(\mathbf{U})$ if and only if $\left.\mathcal{W}(\psi, \Gamma(a, b))\right)$ is a wavelet frame for $H^{2}(\mathbf{U})$. Our next result is an upper bound on the size of the parameters $(a, b)$ (or a lower bound on density) necessary to generate sampling sequences in the true polyanalytic Bergman space, or wavelet frames with Laguerre functions.

The vector valued system $\mathcal{W}(\mathbf{g}, \Lambda)=\left\{T_{a^{j} b k} D_{a^{j}} \mathbf{g}\right\}$ is a wavelet superframe for $\mathcal{H}$ if there exist constants $A$ and $B$ such that, for every $\mathbf{f} \in \mathcal{H}$,

$$
A\|\mathbf{f}\|_{\mathcal{H}}^{2} \leq \sum_{j, k}\left|\left\langle\mathbf{f}, T_{a^{j} b k} D_{a^{j}} \mathbf{g}\right\rangle_{\mathcal{H}}\right|^{2} \leq B\|\mathbf{f}\|_{\mathcal{H}}^{2} .
$$


Superframes were introduced in a more abstract form in [17] and in the context of "multiplexing" in [4. Take the analyzing vector to be $\boldsymbol{\Phi}_{n}=\left(\Phi_{0}, \ldots, \Phi_{n-1}\right)$ Using the identity

$$
\left\langle\mathbf{f}, D_{\mathbf{s}} T_{x} \boldsymbol{\Phi}_{n}\right\rangle_{\mathcal{H}}=s \mathbf{B e r}^{n} \mathbf{f},
$$

we see that $\mathcal{W}\left(\boldsymbol{\Phi}_{n}, \Lambda\right)$ is a wavelet superframe for $\mathcal{H}$ if and only if $\Gamma$ is a sampling sequence for $\mathbf{A}^{n}(\mathbf{U})$.

7.1. Existence of sampling sequences and frames. In this subsection we will prove the existence of wavelet frames with the functions $\Phi_{n}$, provided the hyperbolic lattice is sufficiently dense. We need some notations from [3]. First recall the hyperbolic distance in the half-plane

$$
d\left(z_{1}, z_{2}\right)=\frac{1}{2} \log \frac{1+\rho\left(z_{1}, z_{2}\right)}{1-\rho\left(z_{1}, z_{2}\right)}
$$

where $\rho\left(z_{1}, z_{2}\right)$ is the pseudohyperbolic distance:

$$
\rho\left(z_{1}, z_{2}\right)=\left|\frac{z_{1}-z_{2}}{z_{1}-\overline{z_{2}}}\right|
$$

Given a continuous function $h$ in $\mathbb{R} \times \mathbb{R}^{+}$, we define its local maximal function as

$$
M h(z)=\sup _{w \in B(z, 1)}|h(w)|,
$$

where $B(z, 1)$ is the hyperbolic ball of center $z$ and radius 1 in $\mathbb{R} \times \mathbb{R}^{+}$. Now, define

$$
k_{g}(z)=\left\langle\psi, T_{x} D_{s} g\right\rangle_{H^{2}(\mathbf{U})}, z=x+i s,
$$

and

$$
M B=\left\{g: M k_{g} \in L^{1}(\mathbf{U})\right\}
$$

With these notations, Theorem 4.9 in 3 reads:

Theorem B. Let $W_{g}$ be the wavelet space associated to the analyzing wavelet $g$. If $g \in M B$, then there is a $\delta$ such that every uniformly discrete set $\Gamma$ satisfying $B(z, \delta) \cap \Gamma \neq \varnothing$ for every $z$, is a sampling set for $W_{g}$.

Lemma 2. $\Phi_{n}(z) \in M B$.

Proof. Using (5.2) twice and evaluating the resulting wavelet transforms on the Fourier side, gives:

$$
\begin{aligned}
\left\langle\Phi_{n}, T_{x} D_{s} \Phi_{n}\right\rangle_{H^{2}(\mathbf{U})} & =\sum_{k=0}^{n} \sum_{j=0}^{n}(-2)^{k+j}\left(\begin{array}{l}
n \\
k
\end{array}\right)\left(\begin{array}{l}
n \\
j
\end{array}\right)\left\langle\psi_{j+\frac{1}{2}}, T_{x} D_{s} \psi_{k+\frac{1}{2}}\right\rangle_{H^{2}(\mathbf{U})} \\
& =\sum_{k=0}^{n} \sum_{j=0}^{n}(-2)^{k+j}\left(\begin{array}{l}
n \\
k
\end{array}\right)\left(\begin{array}{l}
n \\
j
\end{array}\right) s^{k+1} \int_{0}^{\infty} t^{1+k+j} e^{(i z-1) t} d t \\
& =\sum_{k=0}^{n} \sum_{j=0}^{n} i^{1+k+j}(-2)^{k+j}\left(\begin{array}{l}
n \\
k
\end{array}\right)\left(\begin{array}{c}
n \\
j
\end{array}\right) \Gamma(1+k+j) \frac{s^{k+1}}{(z+i)^{1+k+j}}
\end{aligned}
$$

Now, since $u_{k, j}(z)=1 /(z+i)^{1+k+j}$ is analytic on the upper half plane, then its maximum on the ball $B(z, \delta)$ is bounded by the average of $u_{k, j}(z)$ on the ball. For this reason, $u_{k, j}(z) \in L^{1}(\mathbf{U})$ implies $u_{k, j}(z) \in M B$. As a result, also $\Phi_{n}(z) \in$ $M B$.

Combining this Lemma with the theorem above, we can assure the existence of Wavelet frames with windows $\Phi_{n}$. 
Theorem 5. It is possible to choose a $\delta>0$ such that every uniformly discrete set $\Gamma$ satisfying $B(z, \delta) \cap \Gamma \neq \varnothing$ for every $\left.z, \mathcal{W}\left(\Phi_{n}, \Lambda\right)\right)$ is a wavelet frame for $H^{2}(\mathbf{U})$.

Corollary 2. It is possible to choose a $\delta$ such that every uniformly discrete set $\Gamma$ satisfying $B(z, \delta) \cap \Gamma \neq \varnothing$ for every $z, \Gamma$ is a sampling sequence for $\mathcal{A}^{n}(\mathbf{U})$.

Corollary 3. It is possible to choose a $\delta$ such that every uniformly discrete set $\Gamma$ satisfying $B(z, \delta) \cap \Gamma \neq \varnothing$ for every $\left.z, \mathcal{W}\left(\Phi_{n}, \Lambda\right)\right)$ is a wavelet superframe for $H^{2}(\mathbf{U})$.

Proof. The arguments we have used in Lemma 2 can be adapted to prove the existence of superframes of this form, since

$$
\left\langle\mathbf{\Phi}_{n}, T_{x} D_{s} \boldsymbol{\Phi}_{n}\right\rangle_{\mathcal{H}}=\sum_{k=0}^{n-1}\left\langle\Phi_{k}, T_{x} D_{s} \Phi_{k}\right\rangle_{H^{2}(\mathbf{U})} \in M B .
$$

7.2. Necessary conditions on the hyperbolic lattice. Now we give necessary conditions for the sampling sequences and frames to exist. Our next result is an upper bound on the size of the parameters $a$ and $b$ (or a lower bound on density) necessary to generate sampling sequences in the true polyanalytic Bergman space, or wavelet frames with the functions $\Phi_{n}$. For this purpose, we will adapt the proof of the necessity part of Theorem 1.1 in [28. An essential step is to associate to $\Gamma(a, b)$ the analytic function

$$
h(z)=\left(\prod_{k=0}^{\infty} \frac{\sin \pi b^{-1} a^{-k}\left(i a^{k}-z\right)}{\sin \pi b^{-1} a^{-k}\left(i a^{k}+z\right)}\right)\left(\prod_{m=1}^{\infty} e^{\frac{2 \pi}{6}} \frac{\sin \pi b^{-1} a^{m}\left(z-i a^{-m}\right)}{\sin \pi b^{-1} a^{m}\left(z+i a^{-m}\right)}\right),
$$

which vanishes in $\Gamma(a, b)$ and plays the role of the sine function in the Paley-Wiener space and of the Weierstrass $\sigma$-function in the Bargmann-Fock space. Following [28, one can check that

$$
h(a z)=-e^{-\frac{2 \pi}{b}} h(z) .
$$

Then, from an estimate of the growth of $h$ on the strip $a^{-1 / 2}<y<a^{1 / 2}$, the following global estimate is obtained:

$$
|h(z)| \leq C s^{-\frac{2 \pi}{b \ln a}} .
$$

Another ingredient in the proof is a result from [3], which gives the stability, with respect to the jittered error, for general wavelet spaces.

Theorem C [3, Theorem 4.4]. Let $W_{g}$ be the wavelet space associated to the analyzing wavelet $g$. If $\Lambda=\left\{z_{j}\right\}$ is a sampling set for $W_{g}$, there exists $\delta>0$ such that if $\Gamma=\left\{w_{j}\right\}$ satisfies $\rho\left(z_{j}, w_{j}\right)<\delta$ for all $j$, then $\Gamma$ is also a sampling set.

Theorem 6. If $z_{m k}=a^{m}(b k+i)$ is a sampling sequence for $\mathcal{A}^{n}(\mathbf{U})$, then

$$
b \log a<2 \pi(n+1) .
$$

Proof. Suppose that $\Gamma(a, b)$ is a sampling sequence. The growth estimate (17.4) gives:

$$
h(z) \in A(\mathbf{U}) \Longleftrightarrow \frac{4 \pi}{b \ln a}<1 .
$$


As a result, if $b \log a>4 \pi(n+1)$, then $h^{n+1}(z) \in A(\mathbf{U})$. Thus, there exists a $f \in L^{2}\left(\mathbb{R}^{+}\right)$such that $h^{n+1}(z)=\operatorname{Ber} f(z)$. Now consider the function

Clearly,

$$
H(z)=\frac{1}{(2 i)^{n} n !}\left(\frac{d}{d z}\right)^{n}\left[s^{n} h^{n+1}(z)\right] .
$$

$$
H(z)=\frac{1}{(2 i)^{n} n !}\left(\frac{d}{d z}\right)^{n}\left[s^{n} \operatorname{Ber} f(z)\right]=\operatorname{Ber}^{n} f(z) \in \mathcal{A}^{n}(\mathbf{U}) .
$$

Since $H(z)$ vanishes on $\Gamma(a, b), \Gamma(a, b)$ cannot be a sampling sequence for $\mathcal{A}^{n}(\mathbf{U})$. It follows that $b \log a \leq 2 \pi(n+1)$.

To prove that the inequality is strict, observe that, by Theorem $\mathrm{C}$, there exists a $\delta>0$ such that if $\Gamma=\left\{w_{m k}\right\}$ satisfies $\rho\left(z_{m k}, w_{m k}\right)<\delta$ for all $m, k$, then $\Gamma$ is also a sampling sequence. Thus, if $b \log a=2 \pi(n+1)$, we can choose $\delta_{0}$ such that $w_{m k}=a^{m}\left(b k+i\left(1-\delta_{0}\right)\right)$ satisfies $\rho\left(z_{m k}, w_{m k}\right)<\delta$ and therefore it is a sampling sequence. This is impossible by the argument in the previous paragraph, since $\left\{w_{m k}\right\}=\Gamma\left(a, b /\left(1-\delta_{0}\right)\right)$ and $b /\left(1-\delta_{0}\right) \log a>b \log a=2 \pi(n+1)$.

Corollary 4. If $\left.\mathcal{W}\left(\Phi_{n}, \Lambda\right)\right)$ is a wavelet frame in $H^{2}(\mathbf{U})$, then

$$
b \log a<2 \pi(n+1) .
$$

Proof. The equivalence between the wavelet frame condition and the sampling condition in the true polyanalytic Bergman space follows from the identity:

$$
\left\langle f, D_{\mathbf{s}} T_{x} \Phi_{n}\right\rangle_{H^{2}(\mathbf{U})}=W_{\Phi_{n}} f(x, s)=s \operatorname{Ber}^{n} \mathcal{F} f(z),
$$

from where it is easily seen that (1.1) and (17.2) are equivalent.

Remark 1. Minor adaptations in the proofs of this paper allow us to introduce a weight in the spaces, by considering the Bergman norm

$$
\int_{\mathbf{U}}|f(z)|^{2} s^{\alpha} d x d s<\infty,
$$

with $\alpha>-1$. In this case, $\Lambda$ is a sampling sequence in the space $\mathcal{A}_{\alpha}^{n}(\mathbf{U})$ if and only if $\mathcal{W}\left(\Phi_{k}^{\alpha}, \Lambda\right)$ is a wavelet frame in $H^{2}(\mathbf{U})$, where $\Phi_{n}^{\alpha}$ is defined by $\mathcal{F} \Phi_{n}^{\alpha}(t)=$ $t^{\frac{1}{2}} l_{n}^{2 \alpha}(2 t)$. A necessary condition for this to happen is:

$$
b \log a<2 \pi \frac{n+1}{\alpha+1} .
$$

Remark 2. Since the superframe property requires every system $\mathcal{W}\left(\Phi_{k}, \Lambda\right)$ to be a frame, it follows that $b \log a<2 \pi$ is a necessary condition for $\mathcal{W}\left(\Phi_{n}, \Lambda\right)$ to be a wavelet superframe.

\section{REFERENCES}

[1] L. D. Abreu, Sampling and interpolation in Bargmann-Fock spaces of polyanalytic functions, preprint.

[2] L. D. Abreu, On the structure of Gabor and super Gabor spaces, preprint.

[3] G. Ascensi, J. Bruna, Model space results for the Gabor and Wavelet transforms, IEEE Trans. Inform. Theory 55 (2009), no. 5, 2250-2259.

[4] R. Balan, Multiplexing of signals using superframes, In SPIE Wavelets applications, volume 4119 of Signal and Image processing XIII, pag. 118-129 (2000).

[5] M. B. Balk, Polyanalytic Functions, Akad. Verlag, Berlin (1991).

[6] G. Bhatt, B. D. Johnson, E. Weber, Orthogonal wavelet frames and vector-valued wavelet transforms. Appl. Comput. Harmon. Anal. 23 (2007), no. 2, 215-234. 
[7] S. Bildea, D. E. Dutkay, G. Picioroaga, MRA super-wavelets. New York J. Math. 11 (2005), $1-19$.

[8] I. Daubechies, "Ten Lectures On Wavelets", CBMS-NSF Regional conference series in applied mathematics (1992).

[9] P. Duren, E. A. Gallardo-Gutiérrez, A. Montes-Rodríguez, A Paley-Wiener theorem for Bergman spaces with application to invariant subspaces, Bull. London Math. Soc. 39 459-466 (2007).

[10] D. E. Dutkay, P. Jorgensen, Oversampling generates super-wavelets. Proc. Amer. Math. Soc. 135 (2007), no. 7, 2219-2227.

[11] H. G. Feichtinger, K. Gröchenig, Banach spaces related to integrable group representations and their atomic decompositions, I, J. Funct. Anal. 86 (2), 307-340 (1989).

[12] H. G. Feichtinger, W Sun, X. Zhou, Two Banach spaces of atoms for stable wavelet frame expansions. J. Approx. Theory 146 (2007), no. 1, 28-70.

[13] Q. Gu, D. Han, Super-wavelets and decomposable wavelet frames. J. Fourier Anal. Appl. 11 (2005), no. 6, 683-696.

[14] K. Gröchenig, "Foundations Of Time-Frequency Analysis", Birkhäuser, Boston, (2001).

[15] K. Gröchenig, Y. Lyubarskii, Gabor frames with Hermite functions, C. R. Acad. Sci. Paris, Ser. I 344, 157-162 (2007).

[16] K. Gröchenig, Y. Lyubarskii, Gabor (Super)Frames with Hermite Functions, Math. Ann. , 345 , no. 2, 267-286 (2009).

[17] D. Han, D. R. Larson, Frames, bases and group representations. Mem. Amer. Math. Soc. 147 (2000), no. 697 .

[18] C. Heil, G. Kutyniok, Density of weighted wavelet frames. J. Geom. Anal. 13 (2003), no. 3, 479-493.

[19] C. Heil, G. Kutyniok, The homogeneous approximation property for wavelet frames. J. Approx. Theory 147 (2007), no. 1, 28-46

[20] O. Hutnik, On the structure of the space of wavelet transforms, C. R. Math. Acad. Sci. Paris 346 , no. 11-12, 649-652, (2008).

[21] O. Hutnik, A note on wavelet subspaces, Monatsh. Math, online published.

[22] G. Kutyniok, Affine density, frame bounds, and the admissibility condition for wavelet frames. Constr. Approx. 25 (2007), no. 3, 239-253.

[23] G. Kutyniok, Affine Density In Wavelet Analysis. Lecture Notes in Mathematics, 1914. Springer, Berlin, 2007.

[24] S. Lang, "SL(2,R)", Springer (1985).

[25] A. K. Ramazanov, Representation of the space of polyanalytic functions as the direct sum of orthogonal subspaces. Application to rational approximations. (Russian) Mat. Zametki 66 (1999), no. 5, 741-759; translation in Math. Notes 66 (1999), no. 5-6, 613-627 (2000).

[26] J. Ramanathan, T. Steger, Incompleteness of sparse coherent states. Appl. Comput. Harmon. Anal. 2 , no. 2, 148-153 (1995).

[27] M. A. Rieffel, Von Neumann algebras associated with pairs of lattices in Lie groups, Math. Ann. 257, 403-418 (1981).

[28] K. Seip, Regular sets of sampling and interpolation for weighted Bergman spaces, Proc. Amer. Math. Soc. 117, no. 1, 213-220 (1993).

[29] K. Seip, Beurling type density theorems in the unit disc, Invent. Math., 113, 21-39 (1993).

[30] W. Sun, Density of wavelet frames. Appl. Comput. Harmon. Anal. 22 (2007), no. 2, 264-272.

[31] N. L. Vasilesvski, On the structure of Bergman and poly-Bergman spaces. Integral Equations Operator Theory 33, no. 4, 471-488, (1999).

CMuC, Department of Mathematics of University of Coimbra, School of Science and Technology (FCTUC) 3001-454 Coimbra, Portugal

E-mail address: daniel@mat.uc.pt

$U R L:$ http://www.mat.uc.pt/ daniel/ 